\title{
Development, Evaluation, and Selection of Advanced Transit Signal Priority Concept Directions
}

\author{
Amer Shalaby and Jinwoo Lee, University of Toronto \\ John Greenough and Stanley Hung, LEA Consulting Ltd. \\ Michael D. Bowie, Fortran Traffic Systems
}

\begin{abstract}
This article presents a process to define the framework for an advanced Transit Signal Priority (TSP) algorithm. For this study, traffic and transit agencies from a broad range of municipalities in Ontario, Canada, provided their views and expertise on various TSP-related issues, including practical needs, design implementation, performance measures, and challenges in developing effective TSP control systems. Based on their inputs as well as the objectives of the project, a set of TSP control concept directions was developed that are characterized with different methodologies and technologies. A listing of selection criteria was also established to evaluate the proposed TSP concept directions. Using these criteria, a ranking and evaluation process was undertaken to select one final TSP control concept that is of interest to potential users of advanced TSP systems. The work described in this article provides a successful example of a process to build consensus among stakeholders for advancing TSP developments.
\end{abstract}

\section{Introduction}

In September 2001, Transport Canada announced a commitment and solicited proposals for the development of products and services that will accelerate the 
growth of ITS knowledge and skills, and promote the uptake and commercialization of ITS technology in Canada. A joint team from the University of Toronto, LEA Consulting Ltd., and Fortran Traffic Systems was awarded a contract for the development of an advanced Transit Signal Priority (TSP) algorithm, which has the potential to be deployed in the field. The objectives of this project can be summarized as follows:

- Develop and evaluate a unique, innovative TSP algorithm, that has the potential to be deployed in the field.

- Facilitate the exchange of knowledge and ideas between the academic research community and the industrial sector during the algorithm development process.

- Provide a means to improve mobility and transportation efficiency.

- Increase operational and regulatory efficiencies for system users and public agencies.

- Encourage the development of products and services that will accelerate the growth of ITS knowledge and skills, and promote the uptake and commercialization of ITS technology.

This article presents a process to develop the framework of the advanced TSP control algorithm as well as the rationale for the selected algorithm approach, rather than describing the developed algorithm itself. Details of the final products of this research can be found elsewhere (Lee et al. 2005, 2006). This article presents various types of TSP control concepts that are characterized with different methodological and technological components. The design of these multilevel TSP concept directions was based on progressive levels of control concepts for the provision of sophisticated TSP control. This research also provides various TSP-related issues from the perspectives of traffic and transit agencies. Professionals from a broad range of municipalities in Ontario, Canada, representing traffic and transit departments, offered their views and expertise on various TSP-related issues, including practical needs, design implementation, performance measures, and the challenges of developing advanced TSP systems. The work described here provides an example of a process to build consensus among stakeholders for advancing TSP developments.

Historical background of TSP is briefly presented in the next section. The cornerstone of most proposed TSP control concepts involves an accurate transit travel time prediction method to support the achievement of more efficient and effec- 
tive signal priority for both transit and traffic. The state-of-the-art in the transit travel time prediction methods is provided next. Following that is a detailed description of the proposed set of TSP control concepts, the used evaluation criteria, and the selection process.

\section{Background}

\section{TSP Measures}

TSP is a signal control strategy that provides preferential treatments to transit vehicles at signalized intersections. Of the various ITS technologies, TSP offers one of the most cost-effective approaches to enhance the effectiveness and efficiency of transit operations. This concept of providing favorable treatments to transit vehicles has evolved since the 1970s through a number of installations in North America and Europe (Evans and Skiles 1970; Courage and Wallace 1977). Early implementations of TSP systems were found inefficient mainly due to the negative impacts on automobiles and on the existing traffic signal operation. Recently, however, several developments were achieved to meet the increasing demand by many agencies for effective TSP operation in response to the growing traffic congestion and its adverse impacts on transit operation. TSP treatments can be classified into four types, which also roughly represent the evolution of TSP and its level of sophistication over the years (Shalaby and Hemily 2004). These types are described briefly below.

Passive Priority. This treatment refers to the very initial methods of TSP, which simply provide adjusted signal timing to accommodate the slower travel speed of transit vehicles due to dynamic characteristics of heavy vehicles as well as the dwell time incurred at stops. Resetting signal coordination plans based on transit travel time, splitting, or the increasing priority phase are typical passive TSP schemes (Wood and Baker 1992). The great advantages of passive priority methods are their relatively low-cost and ease of implementation and operation, since transit detection or communication equipment required to detect the presence of transit vehicles are not necessary. Passive priority becomes most effective with high transit vehicle frequencies, predictable transit travel times, and overall light or moderate traffic volumes (Vincent et al. 1978). However, passive priority may result in unnecessarily significant delays to nontransit vehicles particularly where traffic demand is heavy, since it operates preferential signal timings for transit vehicles even when buses are not present. 
Active Priority. Active priority addresses the critical shortcomings of passive priority by adopting technologies that selectively detect transit vehicles and communicate this information to the traffic controller. Under this scheme, signal priority is given only when transit vehicles are approaching intersections. Typical active priority systems comprise a transit vehicle sensor located upstream of an intersection approach that requests signal priority, a downstream sensor at the intersection stopline that cancels the priority call, and a signal controller. When a transit vehicle is between the upstream and the downstream sensors, the signal controller provides the designated TSP strategies for predetermined durations. Among the various active priority strategies, green extension of the transit phase and early truncation of the nontransit phase are the most widely implemented schemes. Previous studies investigated the efficiency of the various active priority strategies through field tests and the simulation analyses (Ludwick 1975; Benevelli et al. 1983; Boje and Nookala 1996). Active priority has been successful in speeding up transit vehicles along arterial corridors. However, in some instances, transit vehicles may be granted priority when not needed (e.g., vehicle is ahead of schedule or carrying few passengers), resulting in significant delays to nonpriority traffic.

Conditional Active Priority. Recent advances in Intelligent Transportation Systems (ITS) have provided more capabilities to support sophisticated TSP control. Conditional TSP grants priority selectively to transit vehicles that meet certain conditions based on deviation of the vehicle from the schedule or time elapsed since last awarded priority. Conditional TSP requires additional mechanisms for measuring whether the approaching vehicle meets the criteria for granting priority. These may involve an Automated Vehicle Location (AVL) system for measuring schedule adherence and possibly in the future reliable Automated Passenger Counter (APC) systems for measuring transit vehicle occupancy. Recently, conditional active priority has been implemented in several cities (Fehon et al. 2004; Kimpel et al. 2004). Conditional TSP has the potential of limiting buses running ahead of schedule and of mitigating the impacts of unconditional TSP on nonpriority traffic.

Adaptive Priority. Adaptive TSP control refers to a relatively new generation of priority schemes, which seeks to achieve advanced operational objectives by means of adaptive signal control. Examples of operational objectives include improving transit headway regularity, reducing total vehicle delay in the corridor, and maximizing person throughput. Under adaptive TSP, the traffic signal controller adjusts its plan dynamically according to the criteria reflecting the desired objective. Adaptive control often requires feedback of frequently updated traffic and/or 
transit location data into the procedure of signal control adjustment for a better adaptation of rapidly changing traffic and transit situations. Adaptive priority control offers considerable promise for maximizing benefits for both transit vehicles and the general traffic, but the strategy has been only evaluated in laboratory environments and is still in the development stage (Ling and Shalaby 2004; Chang et al. 1998; Conrad et al. 1998).

\section{Transit Travel Time Prediction Models}

The prediction of transit travel times is a critical element in many Advanced Public Transportation System (APTS) applications including Bus Information System (BIS). Many studies have modelled traffic conditions and travel times for automobiles, but only a few have focused on transit travel time prediction. Previous transit arrival prediction efforts are classified into three types according to their adopted techniques, including regression models, Kalman filtering, and neural networks.

Regression Models. As conventional modelling approaches, both linear and nonlinear regression models have been preferably used for transit arrival time prediction because of their relative ease to develop and because they are well suited for parameter estimation problems. Abkowitz and Engelstien (1998) developed two regression-based models to predict mean running time and running time deviation. Some parameters representing the physical bus route characteristics and others representing the dynamic route characteristics were included in the mean running time model, while the running time deviation model was developed in relation to link length and previous running time. Abdelfattah and Khan (1998) also conducted a similar study but the test results of the developed model showed relatively large deviations between the predicted and the actual arrival times.

Kalman Filtering Models. Kalman filtering is a statistical time-series approach, which evolved from state-space representations in linear control theory. Kalman filtering models have relative advantage over other methods in that time-dependent parameters can be included in the model. Wall and Dailey (1999) proposed a Kalman filtering-based transit location tracking model using data obtained from AVL systems. Shalaby and Farhan (2004) demonstrated a Kalman filtering-based dynamic transit travel time prediction model using real-time AVL and APC data. The proposed Kalman filtering model outperformed neural networks and regression models in the simulation analysis.

Artificial Neural Network Models. Artificial Neural Networks (ANNs) have been applied to an increasing numbers of transportation applications over the past 
years. ANNs reproduce the structure and functioning of the brain to mimic its learning capability, which is based on the modification of the connection weight between output and input data. Unlike linear regression methods, ANNs can capture nonlinear relationships between explanatory variables and dependent variables appropriately. Both the studies by Chien et al. (2002) and Kalaputapu and Demetsky (1995) include examples of ANN-based transit arrival prediction models and showed promising test results.

\section{Simulation Models}

Simulation models are very effective tools for analyzing the performance of transportation systems. Real-world systems include interactions among various components, which are very complicated and simultaneously changing, and mathematical modelling approaches are often found inadequate to represent such systems. Simulation models mimic the complicated behavior of systems and provide demonstrations of how those systems are likely to perform. Although simulation models can describe a wide variety of dynamic problems in reality, the applications of simulation have been limited only to off-line applications including analysis, evaluation, and design purposes, mainly due to concerns about the processing time in on-line applications. However, with recent advancements in processing technologies, several studies have adopted on-line simulation modelling for the purposes of traffic prediction (Kosonen and Bargiela 2000) and control (Kosonen 2003). Both studies demonstrated the potential for on-line applications of simulation models. Recently, on-line simulation models for the prediction of transit travel time have been developed for the specific application to TSP (Lee et al. 2005, 2006).

\section{TSP Control Concept Directions}

\section{Development of the Candidate TSP Control Concepts}

To assist the project team in defining a concept direction for an advanced TSP algorithm, a technical advisory group was formed. The group provided technical input throughout the design process, and assisted in the development of concept directions and the final product. The project team invited professionals from a broad range of municipalities in Ontario, Canada, representing traffic and transit agencies that are already operating a TSP program, currently designing a TSP program, or having an interest in creating a TSP program. The strength and benefit of the technical advisory group stemmed from the expertise of some members 
who have operational TSP deployment experience, which could identify and help address real design and operating issues, and who could provide suggestions on potential improvements in TSP operations. Representatives from agencies without current TSP deployments would also provide important input on the needs and features of desired TSP systems for their agencies. The advisory group's composition was generally well balanced between traffic and transit representation. A total of 23 representatives from 10 transit agencies and 13 traffic agencies participated in the project working sessions. Advisory group members were asked about potential issues in TSP from the traffic and transit perspectives. See Table 1 for a summary of their responses.

\section{Table 1. TSP Issues in Traffic and Transit Perspectives}

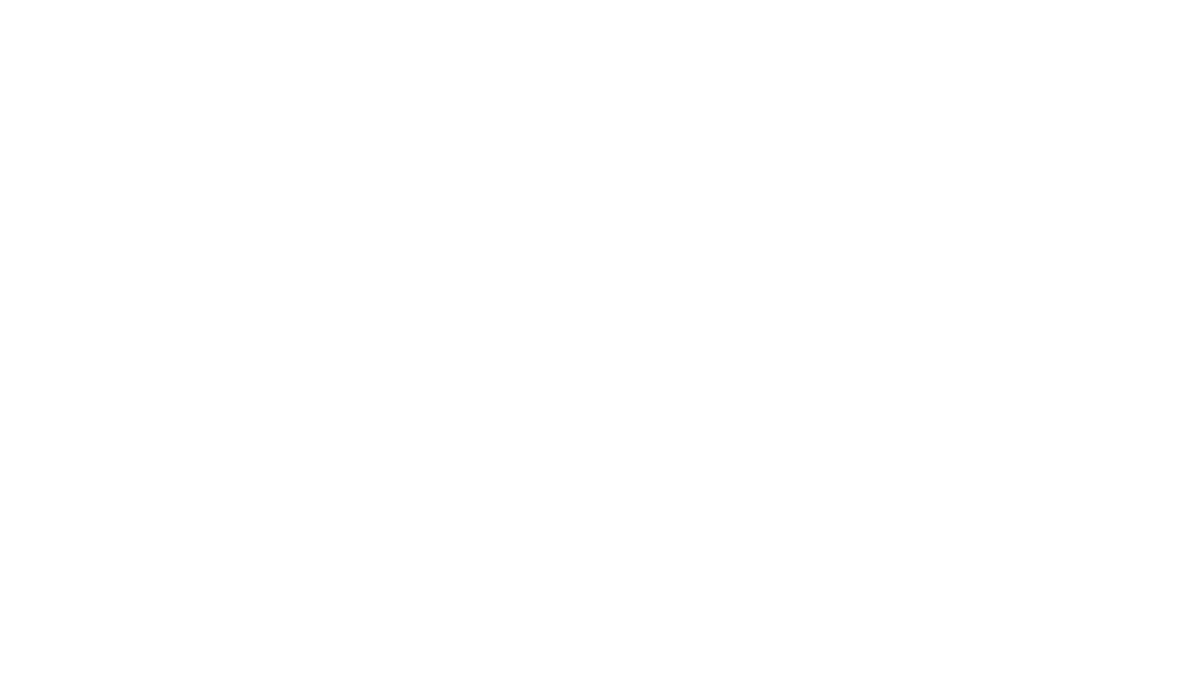

The project team developed a set of multilevel TSP concept approaches based on the collected feedback and also on a literature review of TSP control. The candidate TSP concept approaches are characterized by different methodological and technological components. A higher level TSP concept has the ability to provide more sophisticated TSP control but requires more technologies and equipment than a lower level concept. This multilevel approach design enables the system to built up gradually and to offer varying degrees of TSP control, depending on 
the characteristics of transit and traffic operations. Eight concept directions were developed based on the following assumptions:

- should represent a wide range of possible advancements to active TSP

- should employ an incremental approach to advancement

- assume medium to high frequency service, main transit route, and near-side stops

Figure 1 shows the range of concept directions, while Table 2 provides more details on each concept. The differentiating elements between the concept directions are mainly related to the type of technologies and methodologies used and application context (i.e., single intersection or multiple intersections).

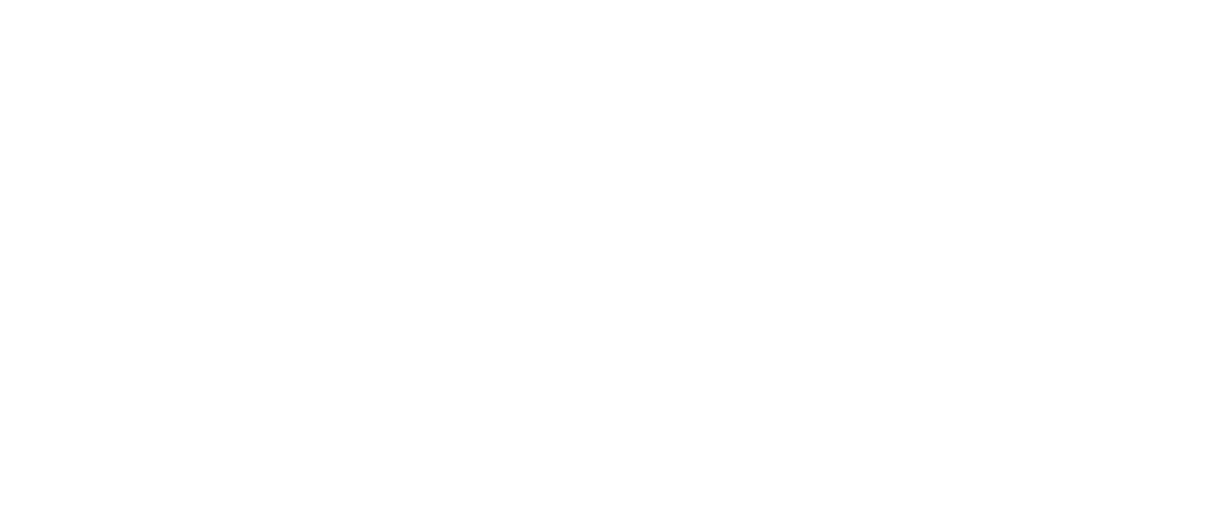

Figure 1. TSP Concept Directions

As shown in Table 2, the developed TSP concept directions include the typical active TSP control method as the most fundamental concept direction, Level I-1, progressing to the transit route-level TSP control as the highest concept (Level III1). Detailed descriptions about the TSP control methods and relative advantages and limitations follow.

Level I-1. The Level I-1 concept direction provides unconditional TSP control using operation rules, which define a "decision point" and TSP strategies. The signal controller actuates TSP strategies, such as green extension and red truncation, at a decision point in the signal cycle if priority is requested. All approaching transit vehicles can request signal priority under operation of this TSP concept. This con- 
Table 2. TSP Concept Directions

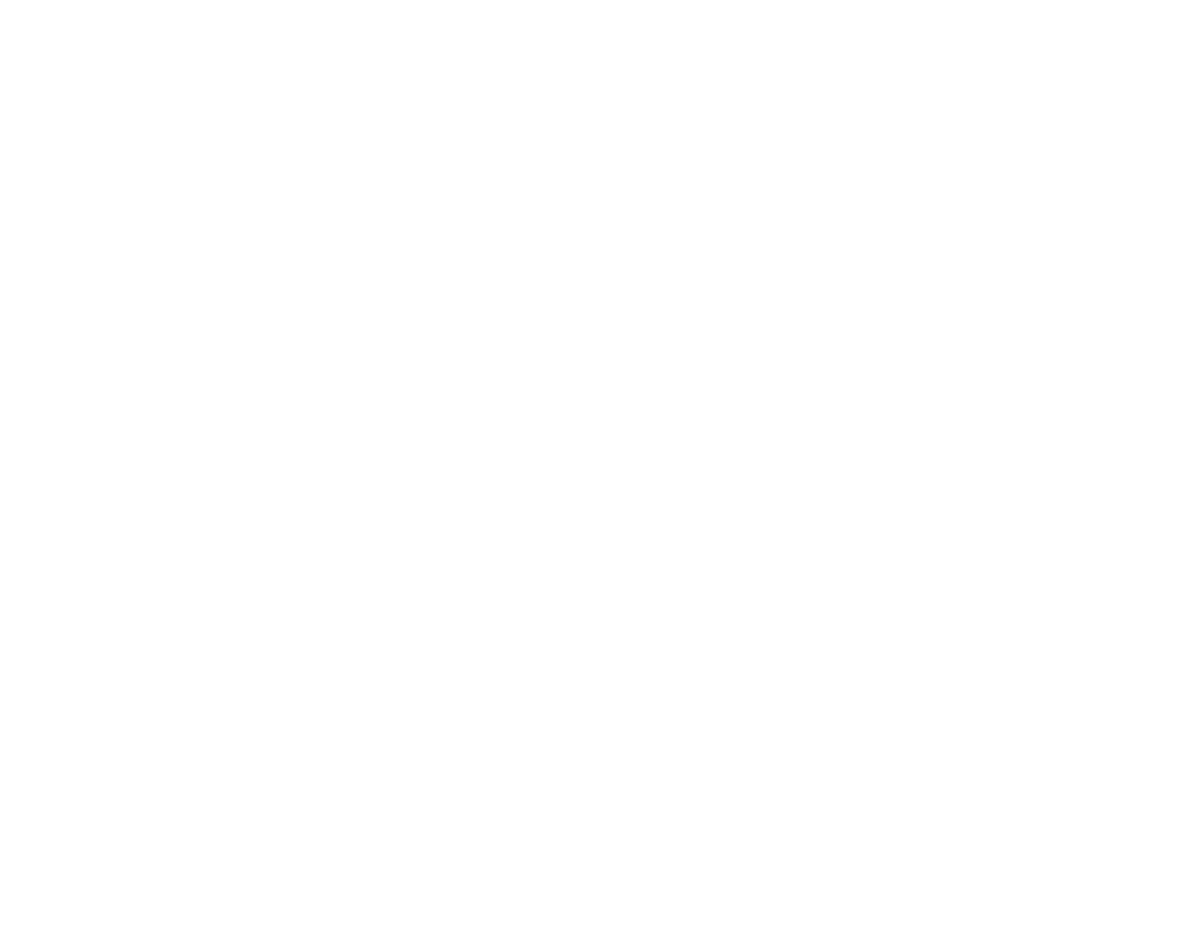

cept direction requires only simple control logic and inexpensive equipment, and has the ability to reduce transit signal delay. However, it also may result in ineffective TSP control, negative impacts on side-street traffic, and unreliable transit service by granting signal priority to transit vehicles even running ahead of schedule.

Level I-2. Level I-2 adopts an improved TSP control rule. The signal controller decides whether to provide signal priority depending on the average transit vehicle arrival time in the signal cycle and the average transit travel time to the stopline. For instance, the controller does not grant the green extension strategy if the detected vehicle is not expected to travel through the intersection by the end of the extended transit phase. This concept direction has a relative advantage compared to Level $\mathrm{I}-1$ by reducing the frequency of signal timing modifications, and consequently lessening negative impacts on side-street traffic. However, the variability in transit travel time may lead to inefficient TSP control particularly where the traffic conditions change unexpectedly and rapidly. 
Level I-3. This concept direction builds on Level I-2 by employing AVL and/or APC equipment to provide conditional signal priority based on the actual headway and/or occupancy of the approaching transit vehicle. Using historical and current AVL data, this concept direction may adopt a simple transit travel time prediction model (e.g., regression). This control concept may avoid providing signal priority to transit vehicles that are ahead of schedule or with low occupancies. More accurate transit travel time prediction also may improve the TSP control efficiency (Koonce et al. 2002). However, this concept direction is still insensitive to the actual traffic conditions, leading to some instances of inefficient applications of TSP strategies. The TSP control in this level (and all Level I concepts) focuses only on transit vehicles at individual intersections. Traffic conditions along cross streets are not considered in all Level l-type concepts.

Level II-1. The distinct difference between Level I and Level II concepts lies in the dynamic transit travel time prediction using real-time traffic and transit sensor data. This enhancement enables the signal controller to operate more TSP strategies such as transit phase early truncation, which demands a high degree of prediction accuracy. Dynamic TSP control methods would select the most appropriate TSP strategy among a number of strategies based on the prediction result. This approach is expected to reduce significantly instances of ineffective operation of TSP strategies bringing about considerable transit delay reduction as well. The Level II- 1 concept employs advanced transit prediction as well as dynamic TSP control as the methodological components. Since this TSP concept does not employ side-street traffic sensors or the AVL system, the TSP control in this level provides unconditional signal priority regardless of transit schedule adherence or side-street traffic conditions.

Level II-2. The Level II-2 concept direction further improves the previous strategy by employing traffic sensors in cross-street approaches. The signal controller has the ability to consider explicitly the side-street traffic condition using the traffic count data or simple traffic flow model when it decides whether to provide TSP strategies. Some threshold values in terms of side-street traffic delay may be defined for the conditional TSP control. The collected traffic sensor data also can be used for different purposes (e.g., to establish traffic operation or management plan). Compared to the more advanced TSP control concepts (i.e., Level II-3, II-4, and III-1), this level of TSP control also does not consider transit vehicles running ahead of schedule and/or that are empty. 
Level II-3. The Level II-3 concept, similar to the Level I-3 concept, uses AVL and/or APC equipment for the transit headway and/or occupancy-based conditional TSP control. However, real-time AVL data also can be used to calibrate the prediction result or to update the transit travel times as the detected transit vehicle approaches the stopline (Lee et al. 2006). Under the operation of this control concept, provision of TSP is conditional on cross-street traffic conditions, schedule adherence, and passenger occupancy. Using transit vehicle location real-time information, this level can obtain improved prediction accuracy. However, it does not necessarily achieve an optimal solution with regard to delay reduction and minimization of impacts. This limitation also applies to all previous concepts that employ a rule-based TSP control method.

Level II-4. The Level II-4 concept direction attempts to find optimal traffic signal timings for both transit and traffic rather than overriding the normal traffic signal with a predefined TSP strategy. A dynamic optimization tool is required such as Genetic Algorithms or Dynamic Programming. Using real-time transit location information and traffic sensor data, the signal controller continuously adjusts the traffic signal timing plan. In fact, this level of TSP control concept works in a similar way to adaptive traffic signal control systems (Robertson and Hunt 1991; Mirchandani and Head 2001; Gartner et al. 2002), except that the transit vehicles are separately considered in the optimization process. The Level II-4 concept offers several advantages over other rule-based signal priority control methods. First, to operate TSP strategies, several parameters (i.e., TSP running signal phases, maximum extension phase length, truncation phase length, etc.) must be predefined for each TSP-operating intersection. The optimization-based TSP control does not need to define such TSP control rules for the optimal TSP operation. Second, in the optimization process, weighting factors can be given to transit and traffic based on a control policy. For instance, more weight can be given to transit vehicle optimization if the control policy is to minimize transit signal delay while maintaining the traffic delay at some level (e.g., during nonpeak time periods). Finally, offset recovery can be operated with more flexibility under the optimization-based control by assigning more signal times to more congested link approaches.

Level III-1. The highest Level III-1 concept expands the application scope of TSP control to transit route or multiintersections. In this level of concept direction, transit vehicle arrival information in the upstream or further intersection link approaches is indicated to downstream intersections through control center or peer-to-peer communications. Downstream signal controllers gradually modify 
traffic signal timing in advance of actual transit vehicle arrival. This prior signal action provides the desired signal phase on transit vehicle arrival and also reduces the negative impacts of sudden traffic signal timing change on other traffic. For this highly sophisticated TSP control, a transit detection system along the transit route is required as well as a long-range transit travel time prediction model. The limitation of this highest level of TSP control lies in the complexities of the required TSP operation software.

\section{Identifying the TSP Concept Evaluation Criteria}

An evaluation method identified the concept directions that are of interest to the stakeholders (e.g., transit service providers, transit users, traffic system operators, and automobile drivers) involved in TSP control. The project team presented the developed TSP concept directions to members of the technical advisory group in a working session. Several questions were posed to the advisory group to identify concept selection criteria that could be used to gauge the relative importance of each concept direction in relation to what is effective and achievable. Table 3 presents responses to the interview questions.

The comments made by each group were recorded and retained by the project team for further consolidation and assessment. The comments would lead to the evaluation and selection of a short list of TSP concept direction candidates for further refinement and evaluation. With the comments and feedback gathered from the working session with the advisory group members, the team reorganized the information into a list of evaluation criteria (see Table 4).

\section{Selection of Viable TSP Concept Directions}

A two-phase ranking methodology was used based on the primary criteria identified by the technical advisory group members as well as the project team. In the first phase of the evaluation, the concepts were ranked, independent of each other, according to the defined criteria. The ranking scale used for this task ranged from 1 to 3; where a value of 1 represented a weak association to the criterion, and a value of 3 represented a high association. Under some criteria it was necessary to use half points to more discretely distinguish between the various concept directions.

In the second phase of the evaluation, each criteria was ranked, independent of each other, according to the general importance of the noted criterion. A ranking scale with values between 1 and 3 was also used; where a value of 1 represented a weaker importance, while a value of 3 represented a greater importance. Values assigned were then multiplied with the respective values determined in phase one 
Table 3. Summary of Responses to Interview Questions

The comments made by each group were recorded and retained by the project team for further consolidation and assessment. The comments would lead to the evaluation and selection of a short list of TSP concept direction candidates for further refinement and evaluation. With the comments and feedback gathered from the working session with the advisory group members, the team reorganized the information into a list of evaluation criteria (see Table 4). 
Table 4. Evaluation Criteria and Factored Ratings

of the evaluation for each concept direction and criteria. The team undertook the ranking and evaluation exercise; the results are shown in Table 5.

Based on this evaluation methodology and the results, it was recommended that the top three ranking concept directions, Levels II-3, II-1, and II-2, be rationalized further through the preliminary design phase of the project.

\section{Preliminary TSP Algorithm Design}

The purpose of the preliminary algorithm design was to further elaborate the definitions and designs of the selected TSP control concepts to help inform the final concept selection process. As part of the preliminary algorithm design phase 
Table 5. Evaluation and Ranking of TSP Concept Directions

of this project, the three top-ranked concept directions were further rationalized, developed, and detailed through an additional working session with the technical advisory group. The intent of this work was to provide further thought and consideration on how the concepts could be physically installed, operated, and controlled. Through this derivation effort, the TSP concepts were discussed in greater detail to settle on a final concept direction to be developed through a detailed design process and eventually tested in a microsimulation environment. 
Level II-1 Concept Design. Figure 2 illustrates the system configuration for the concept Level II-1.

- Transit vehicles are equipped with TSP signal transmitters that are always active.

- TSP detectors, or detection points, are located at the link upstream and the stopline.

- Traffic detectors are located up and down stream along the transit route to measure traffic volumes, speed, and occupancy; data are relayed to the traffic signal controller.

- Traffic signal controller will assess the data through a travel time prediction model with real-time transit travel time and traffic data as inputs.

- TSP strategies are initiated by the traffic signal controller based on the predicted transit vehicle travel time through a rule-based algorithm.

- TSP sequence will be unconditionally provided.

- Traffic signal controller would issue a signal timing recovery plan after the TSP call is dropped or maxed out.

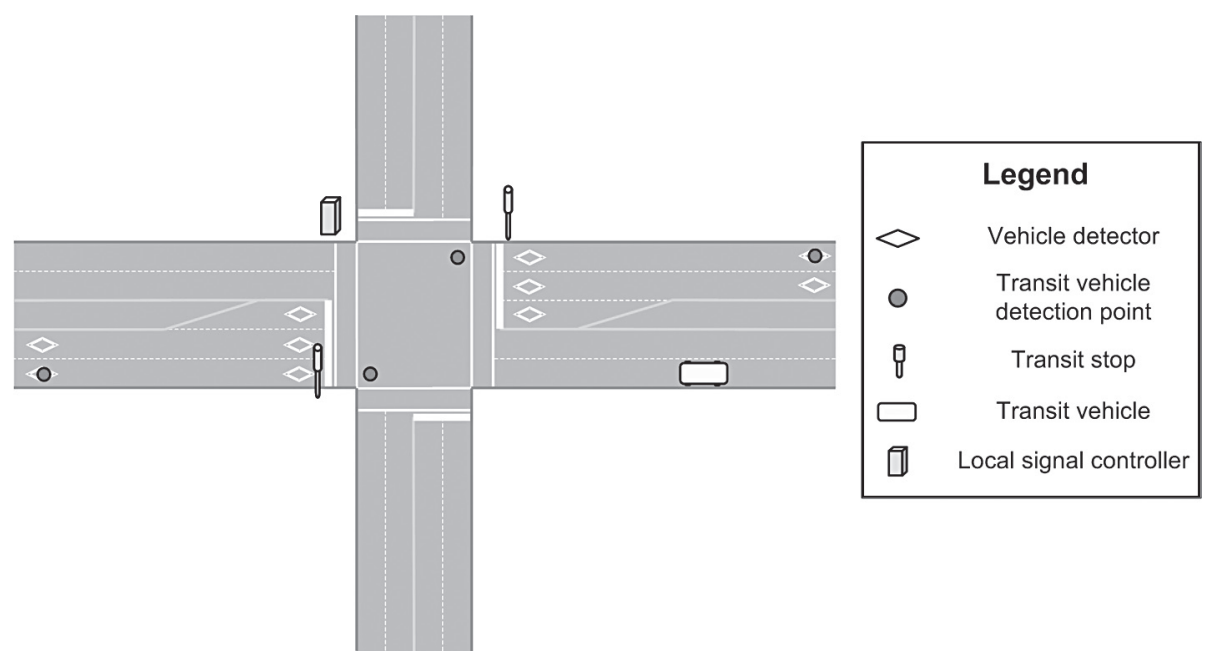

Figure 2. Level II-1 Concept Configuration 
Level II-2 Concept Design. The system configuration for the Level II-2 concept direction is given in Figure 3.

- Transit vehicles are equipped with TSP emitters that are always active.

- Traffic detectors are located up and down stream along the transit route and also in the cross- street approaches to measure traffic volumes, speed, and occupancy; data are relayed to the traffic signal controller.

- Implementation of the TSP strategy will be conditional based on the overall effect on cross-street traffic.

- TSP strategies are initiated by the traffic signal controller based on the predicted transit vehicle travel time through a dynamic rule-based algorithm.

- Traffic signal controller would issue a signal timing recovery plan after the TSP call is dropped or maxed out.

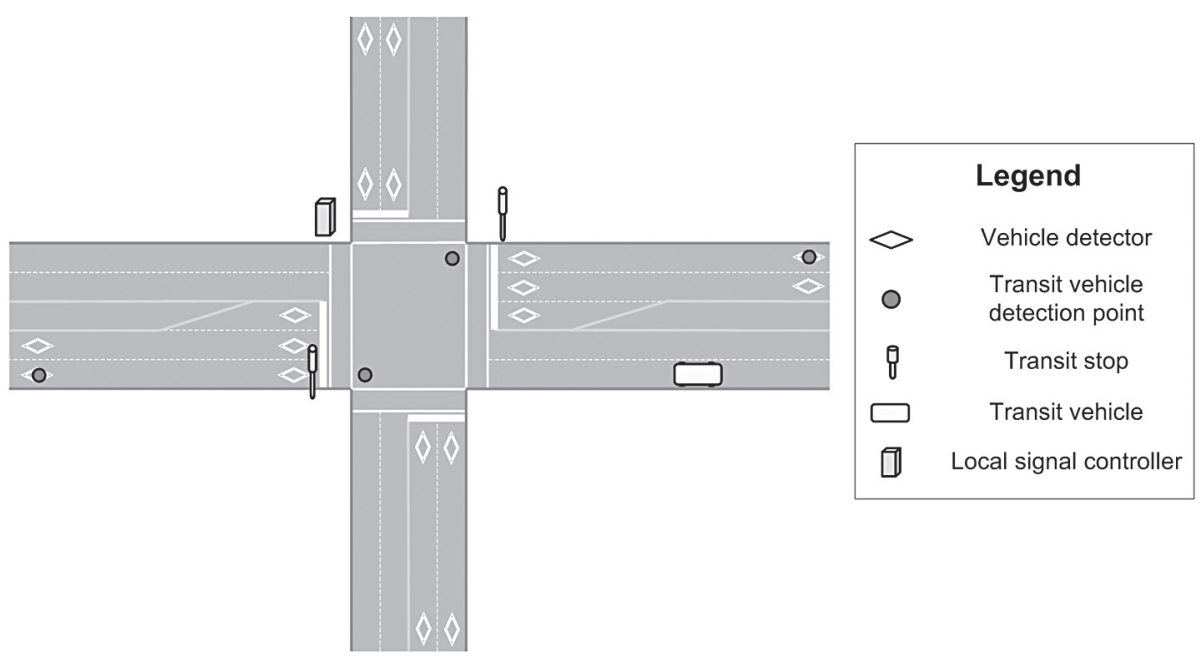

Figure 3. Level II-2 Concept Configuration

Level II-3 Concept Design. Figure 4 depicts the Level II-3 concept configuration.

- Transit vehicles are equipped with an intelligent computational device such as vehicle logic unit (VLU).

- On-board AVL system provides VLU with real-time position data. 
- VLU determines if TSP is required through a rule-based algorithm associating schedule adherence and/or vehicle occupancy.

- Traffic signal controller determines the predicted travel time through AVL-gathered data; prediction model could also reference historical route travel data.

- If the VLU determines that a TSP call is warranted to maintain the transit schedule, the TSP emitter would be activated at the desired point along the route.

- Traffic detectors are located up and down stream along the transit route and in the cross-street approaches to measure traffic volumes, speed, and occupancy; data are relayed to the traffic signal controller.

- Traffic signal controller will assess the data through a travel time prediction model with real-time AVL transit travel time and traffic data from all approaches as inputs.

- Traffic signal controller continuously updates the predicted arrival time of the transit vehicle.

- Implementation of the TSP strategy will be conditional based on the overall effect on cross-street traffic.

- TSP strategies are initiated by the traffic signal controller based on the predicted transit vehicle arrival time through a rule-based algorithm.

- Traffic signal controller would issue a signal timing recovery plan after the TSP call is dropped or maxed out. 


\section{Selection of the Final TSP Concept Direction}

The preliminary designs of the three short-listed concept directions were presented to the technical advisory group, and feedback was solicited. Most members responded that the selected concept directions were within their expectations, and provided some recommendations related to further development.

- In selecting the final concept, the required policies, functionality, and g/C ratios should be taken into consideration.

- The validity of the concept (i.e., ability to monitor the performance) should be addressed.

- Complex systems are not necessarily good for small municipalities. Can the costs be justified?

- Criteria for the deployment of each concept must be developed and defined. Where and when could the concept be used?

- Clear public policy objectives must be identified.

Based on the preliminary designs and on the additional feedback received from the technical advisory group, the project team discussed which of the preferred concept directions to move forward into detailed design, modelling, simulation, and evaluation as a prototype of an advanced TSP algorithm. From a technical standpoint, the project team determined that design and deployment barriers associated with any of the three selected concepts are manageable. Each of the concepts is also an improvement on the status quo deployment and operation of TSP. Therefore, there is no underlying benefit to select one concept over another from a technical outlook.

In considering the three concept directions from a practical deployment perspective, discussion was raised regarding the feasibility of deploying side-street traffic sensors at all intersections. This is surely not a feasible consideration, especially at intersections with low side-street traffic volumes and operating at a good level of service. Therefore, the need for a traffic sensor on the side street should be considered in greater detail (i.e., cost/benefit assessments) during the design phase for each intersection. At the conclusion of the selection process, the team resolved to design and model a variation of the concept direction Level II-3. The variation would exclude the side- street traffic detectors, so the final concept direction does not provide side-street conditional TSP control, while keeping the other features of the Level II-3 concepts. The detailed description of the development of the final 
control concept together with the evaluation results are available elsewhere (Lee et al. 2005, 2006).

\section{Summary}

This article presents an approach to develop a framework for advanced TSP control algorithms. A full range of TSP concept directions, which are defined on the basis of the most critical factors and features, are provided in the research. For this study, traffic and transit agencies from a broad range of municipalities in Ontario, Canada, provided their views and expertise on various TSP-related issues including practical needs, design implementation, performance measures, and challenges in developing effective TSP systems. Based on the technical inputs, the project team developed a multilevel framework for TSP concept directions that provide different levels of sophistication for TSP control. Three TSP control concepts were selected for further development following an evaluation and ranking process. All of the selected concept directions included a component of transit travel time prediction, which certainly indicates the demand for more efficient TSP control method. Further detailed design and configuration information were prepared and presented to the technical advisory group members for the selection of the final TSP control concept. Based on the additional feedback received from the technical advisory group and the feasibility of deployment, the Level II-3 concept direction with a minor variation was selected as a prototype of an advanced TSP control algorithm. Detailed information about the developed advanced TSP control algorithm can be found elsewhere (Lee et al. 2005, 2006). Finally, the work described in this article provides a successful example of a process to build consensus among stakeholders for advancing TSP developments.

\section{Acknowledgment}

This research has been supported by Transport Canada under the ITS R\&D program.

\section{References}

Abkowitz, M. D., and I. Engelstien. 1983. Factors affecting running time on transit routes. Transportation Research 17A (2): 107-113. 
Abdelfattah, A. M., and A. M. Khan. 1998. Models for predicting bus delay. Transportation Research Record 1623: 8-15.

Benevelli, D. A., A. E. Radwan, and J. W. Hurley. 1983. Evaluation of a bus preemption strategy by use of computer simulation. Transportation Research Record 906: 60-67.

Boje, B.F., and M. Nookala. 1996. Signal priority for buses: An operational test at Louisiana Avenue, Minneapolis. Compendium of Technical Papers for the 66th ITE Annual Meeting. Washington DC: 309-313.

Chang, G. L, M. Vasudeva, and C. C. Su. 1998. Bus preemption under adaptive signal control environments. Transportation Research Record 1494: 146-154.

Chien, S. I., Y. Ding and C. Wei. 2002. Dynamic bus arrival time prediction with artificial neural networks. Journal of Transportation Engineering 128 (5): 429-438.

Conrad, M., F. Dion, and S. Yagar. 1998. Real-time traffic signal optimization with transit signal priority. Transportation Research Record 1634.

Courage, K. G., and C. E. Wallace. 1977. Evaluation of some bus priority strategies on NW 7th Avenue in Miami. Transportation Research Record. 626: 32-35.

Evans, H., and G. and Skiles. 1970. Improving public transit through bus preemption of traffic signals. Traffic Quarterly 24 (4): 531-543.

Fehon, K., J. Jarzab, C. Emoto, and D. Dagang. 2004. Transit signal priority for Silicon Valley bus rapid transit. DKS Associates, http://www.dksassociates.com/, Accessed July.

Gartner, N. H., F. J. Pooran, and C. M. Andrews. 2002. Optimized policies for adaptive control strategy in real-time traffic adaptive control systems: Implementation and field testing. Transportation Research Record 1811: 148-156.

Kalaputapu, R., and M. Demetsky. 1995. Modeling schedule deviations of buses using AVL data and artificial neural networks. Transportation Research Record 1497: 44-52.

Koonce P., J. Ringert, T. Urbanik, W. Rotich, and B. Kloos. 2002. Detection range setting methodology for signal priority. Journal of Public Transportation 5 (2): 113-136. 
Kosonen, I., and A. Bargiela. 2000. Simulation-based traffic information system. 7th World Congress on Intelligent Transport Systems. Turin, Italy.

Kosonen, l., 2003. Multi-agent fuzzy signal control based on real-time simulation. Transportation Research 11C: 389-403

Kimpel, T. J., J. Strathman, R. Bertini, P. Bender, and S. Callas. 2004. Analysis of transit signal priority using archived TriMet bus dispatch system data. 83rd Annual Meeting of the Transportation Research Board, Washington, DC.

Lee, J., B. Shalaby, J. Greenough. M. Bowie, and S. Hung. 2005. Advanced transit signal priority control using on-line microsimulation-based transit prediction model. Transportation Research Record 1925: 185-194.

Lee, J., A. Shalaby, J. Greenough, M. Bowie, and S. Hung. 2006. TSP-advance: An advanced transit signal priority system-Development and evaluation. 85th Transportation Research Board Annual Meeting, Transportation Research Board, National Research Council, Washington, DC.

Ling, K., and A. Shalaby. 2004. Automated transit headway control via adaptive signal priority. Special Issue on Modeling for Transit Operations and Service Planning, Journal of Advanced Transportation 38 (1): 45-67.

Ludwick, J. S. 1975. Simulation of an unconditional preemption bus priority system. Transportation Research Record 906: 1-10.

Mirchandani, P., and L. Head. 2001. A real-time traffic signal control system: Architecture, algorithms, and analysis. Transportation Research 9C: 415-432.

Robertson, D.I., and P. B. Hunt. 1991. Optimizing networks of traffic signals in real time-The SCOOT method. IEEE Transactions on Vehicular Technology 40 (1).

Shalaby, A., and A. Farhan. 2004. Prediction model of bus arrival and departure time using AVL and APC data. Journal of Public Transportation 7 (1): 41-61.

Shalaby, A., and B. Hemily. 2004. Urban public transit ITS research and development in Canada. Work Group 1.3, ATLANTIC Project.

Vincent, R.A., B. R. Coope, and K. Wood. 1978. Bus-actuated signal control at isolated intersections. Report 814. Crowthorne, UK: Transportation and Road Research Laboratory. 
Wall, Z., and D. J. Dailey. 1999. An algorithm for predicting the arrival time of mass transit vehicle using automatic vehicle location data. 78rd Annual Meeting of the Transportation Research Board, Washington, DC.

Wood, K., and R. T. Baker. 1992. Using SCOOT weightings to benefits strategic routes. Traffic Engineering and Control: 226-235.

\section{About the Authors}

AMER ShALABY (amer@ecf.utoronto.ca) received his master's and Ph.D. degrees from the Department of Civil Engineering, University of Toronto in 1991 and 1997, respectively. He was a postdoctoral fellow at the university's Joint Program of Transportation in 1996-1997 and an NSERC Industrial Research Fellow at IBI Group in 1997-1998. From 1998-2000, he was an assistant professor at Ryerson University, where he also directed the Vehicle Safety Research Centre. Since January 2001, Dr. Shalaby has been an assistant professor at the University of Toronto. He research specialty is in the area of ITS, with emphasis on transit applications. His research interests also include strategic and service planning of public transit systems.

JINwoo LeE (leeji@ecf.utoronto.ca) is a Ph.D. student and a graduate student researcher in the Department of Civil Engineering at the University of Toronto. His research areas of interest include traffic signal control, transit signal priority, and ITS applications. Mr. Lee is also a member of the Transportation Systems Group of LEA Consulting Ltd., providing his technical expertise on various traffic engineering and ITS-related projects.

JOHN GREeNOUGH (jgreenough@LEA.ca) is vice president of LEA Consulting Ltd., Markham, Ontario, and is also head of LEA's Transportation Systems Group. For more than 25 years, Mr. Greenough has been deeply involved in the planning, design, deployment, and operations of many ITS installations across Canada and in the United States, with focus on advanced traffic signal control systems, freeway and corridor traffic management systems, advanced transit management systems, including TSP, as well as transportation infrastructure security design services. For nearly a decade, he was a manager of Traffic Systems for the former Metropolitan Toronto, where he directed significant advancements in TSP, along with a complete modernization of much of Toronto's traffic systems. 
MiChael D. Bowie (mbowie@fortrantraffic.com), who was introduced to the traffic control industry in 1967 with Canadian General Electric, has been involved with the design, production, installation, maintenance, and training of numerous ITS products including traffic controllers, video incident detection, and central computer systems. In his current position as manager of Business Development, Mr. Bowie is instrumental in defining customer requirements in the continued product development of Fortran's Fastracs Traffic Management System. These requirements include areas such as TSP, light rail transit preemption and advanced video management systems for incident detection and data collection.

StANLey HUNG (shung@LEA.ca) is a member of the Transportation Systems Group of LEA Consulting Ltd. He has been involved with several TSP and traffic signal control related projects. In respect to state-of-the-art development of TSP, Mr. Hung was part of the project team for the research and development of an advanced TSP algorithm with Transport Canada. Mr. Hung has provided his expertise for the deployment of TSP in the City of Peterborough, the Regional Municipality of York, the City of Edmonton, the City of Brampton, and the City of Toronto. 